Ma. Joan Nemis Nalangan, MD

Department of Otorhinolaryngology Head and Neck Surgery

Armed Forces of the Philippines Medical Center
Correspondence: Dr. Ma. Joan Nemis Nalangan Department of Otorhinolaryngology Head and Neck Surgery Armed Forces of the Philippines Medical Center 7th Floor, Armed Forces of the Philippines Medical Center,

V. Luna Avenue, Quezon City 0840

Philippines

Phone: (632) 4262701 Locals 6172 / 8972

Email:ent_afpmc@yahoo.com

Reprints will not be available from the author.

The author declared that this represents original material that is not being considered for publication or has not been published or accepted for publication elsewhere in full or in part, in print or electronic media; that the requirements for authorship have been met by the author, and that the author believes that the manuscript represents honest work.

Disclosures: The author signed a disclosure that there are no financial or other (including personal) relationships, intellectual passion, political or religious beliefs, and institutional affiliations that might lead to a conflict of interest.

Presented at NEMEC Annual Residents'Interesting Case Contest, 24 March 2011, Valdes Hall, Veterans Memorial Medical Center and PSO-HNS Annual Convention Free Paper Presentation, 04 December 2012, Sofitel Plaza Hotel.

\title{
A Vocal Cord Polyp in a Pediatric Patient
}

\begin{abstract}
Objective: To present a case of vocal cord polyp in a pediatric patient and discuss its differential
\end{abstract} diagnosis, assessment and management.

\section{Methods:}

Design: Case Report

Setting: Tertiary Public Hospital

Patient: One

Results: A seven-year-old girl presented with hoarseness and a benign, unilateral mass seen at the junction of the anterior and middle third of the vocal cord. The hoarseness resolved after excision and histopathology confirmed an inflammatory vocal cord pseudo polyp.

Conclusion: Vocal cord polyps occur infrequently in children and adolescents. These cases are seldomly seen and reported and may arise from chronic abuse of the larynx, vocal cord trauma or phonotrauma. Hoarseness is the most common presenting symptom. Differential diagnoses include recurrent respiratory papillomatosis, laryngeal cyst and laryngeal nodule. The management of vocal cord polyps involves surgical removal followed by speech therapy.

Keywords: hoarseness, phonotrauma, vocal cord polyp, recurrent respiratory papillomatosis, vocal cord nodule, vocal cord cyst, flexible laryngoscopy, speech therapy

\section{CASE REPORT}

A 7-year-old girl from Bulacan, Philippines was referred to us for hoarseness and stridor. The history of present illness started three months prior to admission when she swallowed a fish spine resulting in minimal bleeding from the throat. No foreign body sensation, dysphagia, odynophagia, frequent coughing, difficulty in breathing or associated symptoms were noted and no medical consult was made. A week after the incident, the child experienced hoarseness and occasional dysphagia. No cough, colds or fever were noted. A pediatrician prescribed unrecalled medications which afforded no relief. Two months prior to admission, the patient developed stridor and difficulty in breathing on supine position. Follow up consult with the same pediatrician resulted in treatment for bronchial asthma which only relieved the difficulty of breathing. One month prior to admission, due to persistent hoarseness and stridor, the girl was brought to another pediatrician who again managed her as a case of bronchial asthma. Due to persistence of symptoms, she was finally referred to us.

On examination, flexible laryngoscopy revealed a white pedunculated mass on the right vocal cord. It sucked-in on inspiration but popped out of the glottic area during phonation or expiration. (Figure 1)

She was admitted on intravenous hydrocortisone and oral cetirizine, and she underwent direct laryngoscopy with excision under general anesthesia. (Figure 2) She tolerated the procedure well and stridor resolved.

Initially, there was no noticeable improvement in voice quality despite the absence of residual vocal cord mass on pre-discharge flexible laryngoscopy. (Figure 3) However improvement in voice quality and smooth breathing were noted on follow-up consultation after a week. The histopathology report was consistent with inflammatory pseudopolyp. (Figure 4) 


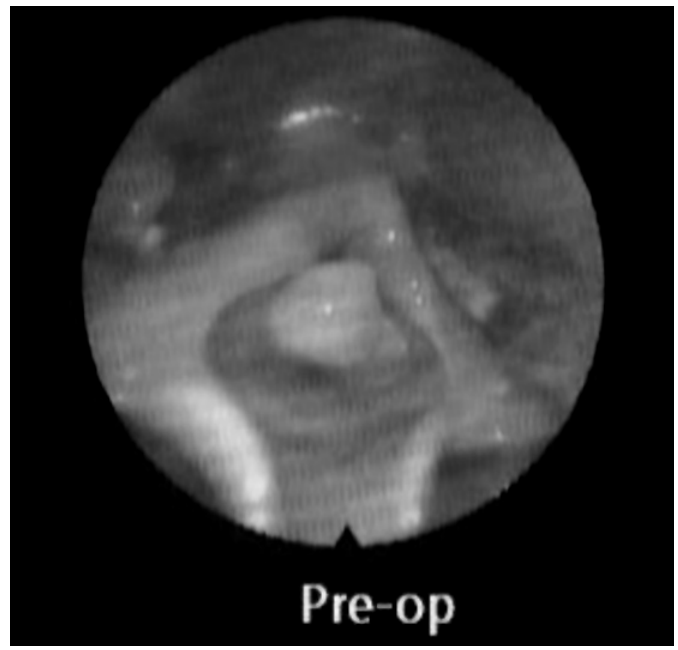

Figure 1. Preoperative flexible laryngoscopy showing a whitish, pedunculated mass occupying the middle third of right true vocal cord that sucked in on inspiration and protruded during expiration or phonation

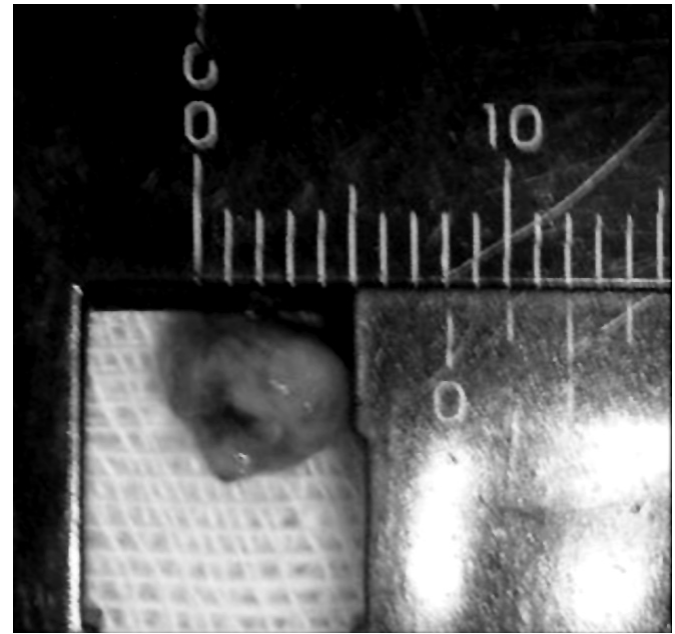

Figure 2. Gross specimen showing a $0.5 \times 0.5 \mathrm{~cm}$, white, round, firm mass attached to and excised from the right true vocal cord

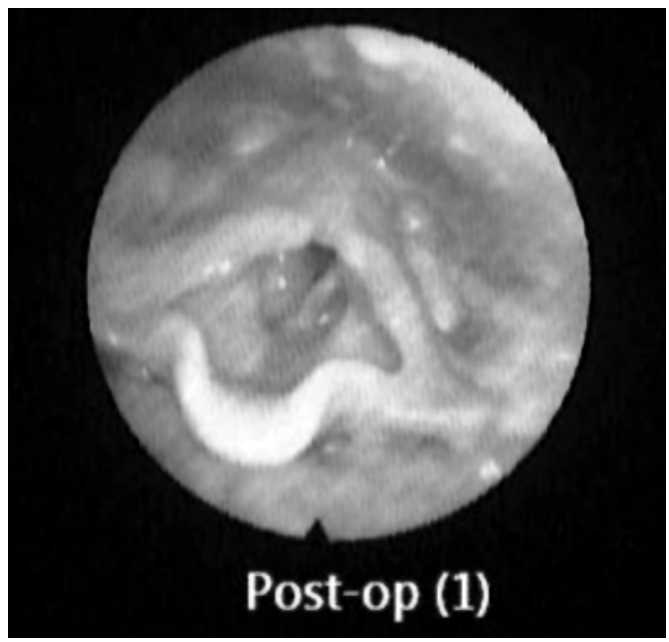

Figure 3A. Pre-discharge flexible laryngoscopy with no residual vocal cord mass

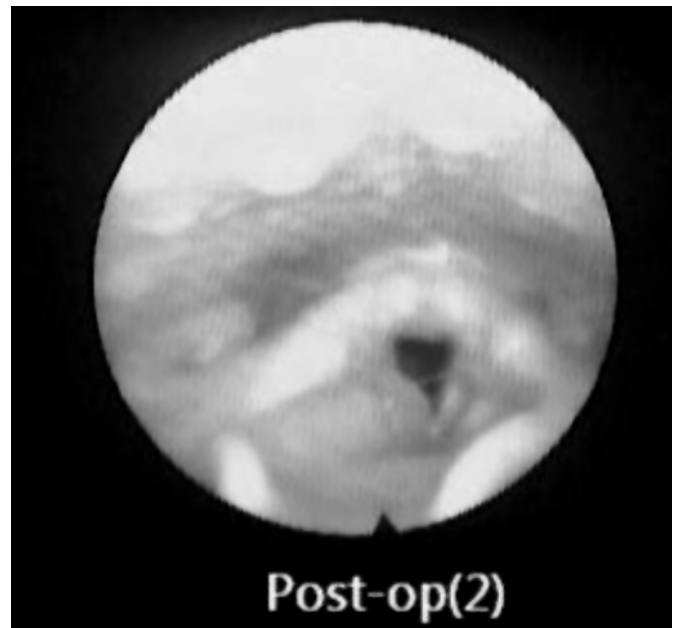

Figure 3B. One week follow-up laryngoscopy with improved voice quality and smooth breathing

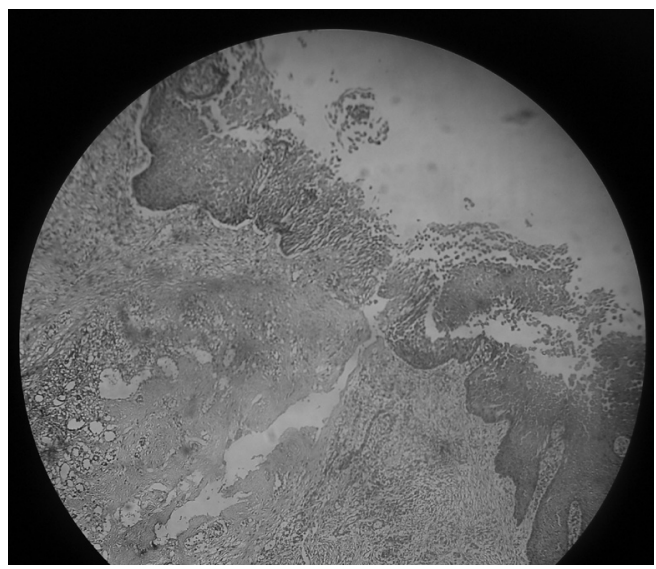

Figure 4. Histopathologic section, Hematoxylin and Eosin (25x) showing overlying benign stratified squamous epithelium with underlying edematous stroma infiltrated by inflammatory cells (lymphocytes, plasma cells, histiocytes and occasional neutrophils) with several congested small blood vessels

\section{DISCUSSION}

Most surveys of children show a $6 \%$ to $9 \%$ incidence of voice disorders. ${ }^{1}$ The underlying cause can be organic or functional. Functional disorders are caused by emotional or psychological problems such as personality disorder, anxiety or adjustment but can lead to anatomic alterations of the vocal cords. ${ }^{2}$ Organic voice disorders result from congenital or acquired anatomic abnormalities which include laryngeal papilloma, web, stenosis, malignant tumor, polyps, nodules and cysts.

Hoarseness is a voice disorder characterized by altered vocal quality, pitch, loudness or vocal effort that impairs communication or reduces voice-related quality of life. It is a common complaint in children of all ages. Among children, prevalence rates vary from $3.9 \%$ to $23.4 \%$, the most affected age range is between 8 to 14 years old. ${ }^{3}$ Most cases related to viral upper respiratory tract infections are self-limited and require no special treatment. Other common causes include 


\section{CASE REPORTS}

vocal nodules, allergic or infectious laryngitis and laryngitis from gastroesophageal reflux. Occasionally, hoarseness heralds the onset of upper airway obstruction or may be the presenting symptom of serious systemic illness and thus warrants a more thorough evaluation and timely, appropriate therapy. ${ }^{4}$

Based on presenting symptoms and endoscopy, differential diagnoses of this case include respiratory papillomatosis, vocal cord cyst and nodule.

Recurrent respiratory papillomatosis is the most common benign neoplasm of the larynx in children and is the second most frequent cause of childhood hoarsenes ${ }^{5,6}$ It is of viral etiology. For this patient, it was ruled out based on the clinical assessment. The patient had hoarseness and stridor but there was no respiratory distress, tachypnea, decreased air entry, cyanosis or other symptoms that may indicate impending respiratory collapse which are usually present in recurrent respiratory papillomatosis. The documented lesion in this patient also differed from the characteristic multiple warty excrescences on the mucosal surface of the respiratory tract typical of papillomatosis.

Vocal cord cysts are subepidermal epithelial-lined sacs located within the lamina propria, and may be mucus retention or epidermoid in origin. Mucus retention cysts form when a mucous gland duct becomes obstructed (usually during an upper respiratory infection or with overuse) retaining glandular secretions. ${ }^{7}$ Histopathology ruled out this entity.

Vocal cord nodules are typically bilateral swellings of the midportion of the membranous vocal folds or may occur at the free edge of anterior and middle third of vocal cord. They are of variable size and are characterised histologically by thickening of the epithelium with a variable degree of inflammation in the underlying superficial lamina propria. ${ }^{8}$ The prevalence of nodules in the general population is not known but it has been reported as being the cause of hoarseness in up to $23.4 \%$ of children. ${ }^{9}$ The etiology of vocal nodules is not known but traditionally they are thought to be due to 'voice abuse' and psychological factors especially in children. Other medical conditions such as infection, allergy and reflux may also play a role in the etiology. ${ }^{10}$ Among the differential diagnoses mentioned, vocal cord nodules were most likely but histopathology ruled it out.

Vocal cord polyps as mentioned, occur infrequently in children and adolescents. ${ }^{11,8}$ They are usually seen at the junction of the anterior and middle third of the vocal cord and may be fusiform, pedunculated or generalized. ${ }^{12}$ The pathophysiology is believed to be attributable to breakage of a capillary in Reinke space (superficial lamina propia) with subsequent extravasation of blood, resultant local edema and ultimate organization with hyalinized stroma. ${ }^{7}$ Polyps are believed to result from phonotrauma; however, they are also recognized to potentially arise from a single episode of hemorrhage. It was found that the combination of signs of recent bleeding and depositions of fibrin and iron pigment in macrophages resided almost exclusively in polyps when compared with other benign lesions. ${ }^{13}$

Morphological analyses of vocal cord polyps show lamina propria with edema, vessel proliferation and inflammation; basement membrane with adhesion loss in some areas; and a dense network of subepithelial collagen. ${ }^{14}$ In our patient, the histopathologic report noted pieces of tissue with overlying benign stratified squamous epithelium. The underlying stroma was edematous, infiltrated by inflammatory cells, consisting of lymphocytes, plasma cells, histiocytes and occasional neutrophils. Several congested small blood vessels are also seen with no cellular atypia. Histopathology confirmed the diagnosis of laryngeal polyp. Although the single episode of trauma specifically ingestion of fish spine was not properly documented, the patient was well prior to this event. The possibility that this was the etiology should be considered and reported, hence this case report.

Vocal cord polyps occur infrequently in children and adolescents. These cases are seldomly seen and reported and may arise from chronic abuse of the larynx, vocal cord trauma or phonotrauma. Hoarseness is the most common presenting symptom. Differential diagnoses include recurrent respiratory papillomatosis, laryngeal cyst and laryngeal nodule. Although most voice disorders in children result from vocal cord nodules, we should also consider vocal cord polyps in a hoarse patient. Hoarseness in children should not be ignored since vocal cord polyps may cause partial airway obstruction and may need immediate management. Hoarse patients should be evaluated by an otolaryngologist and direct visualization must be done even in children. The management of vocal cord polyps involves surgical removal followed by speech therapy. ${ }^{1,12,15}$

\section{REFERENCES}

1. Wilson DK. Management of voice disorders in children and adolescents, Semin Speech Lang Hear. 1983; 4:245-258.

2. Toohill R. The Psychosomatic aspects of children with vocal nodules. Arch Otolaryngol.1975 Oct; 101 (10):591-5

3. Schwartz SR, Cohen SM, Dailey SH, Rosenfeld RM, Deutsch ES, Gillespie MB. et al. Clinical practice guideline: hoarseness (dysphonia). Otolaryngol Head Neck Surg. 2009 Sep; 141 (3 Suppl 2): S1-S31.

4. Chang K, Inglis A. . Evaluation and management of hoarseness in children: Curr Opin Oto Head Neck Surg. 1996 Dec; 4:396-400

5. Gallagher TQ, Derkay CS. Recurrent respiratory papillomatosis: update 2008, Curr Opin Otolaryngol Head Neck Surg. 2008 Dec; 16(6):536-542

6. Morgan AH, Zitsch RP. Recurrent respiratory papillomatosis in children: a retrospective study of management and complications. Ear Nose Throat J. 1986 Sep; 65 (9):19-28.

7. Altman KW. Vocal fold masses. Otolaryngol Clin North Am. 2007 Oct; 40 (5):1091-108.

8. Nagata K, Kurita S, Yasumoto S, Maeda T, Kawasaki H, Hirano M. Vocal fold polyps and nodules A 10-year review of 1,156 patients. Auris Nasus Larynx 1983; 10 Suppl:S27-35.

9. Silverman EM. Incidence of chronic hoarseness among school-age children. J Speech Hear Disord. 1975 May; 40 (2):211-5.

10. McHugh-Munier C, Scherer KR, Lehmann W, Scherer U. Coping strategies, personality, and voice quality in patients with vocal fold nodules and polyps. J Voice. 1997 Dec; 11 (4):452-61.

11. Kambic V, Radsel Z, Zargi M, Acko M. Vocal cord polyps: incidence, histology and pathogenesis. J Laryngol Otol. 1981 Jun; 95 (6):609-18.

12. Kleinsasser O. Pathogenesis of vocal cord polyps. Ann Otol Rhinol Laryngol. 1982 Jul-Aug; 91 (4 pt1):378-81.

13. Dikkers FG, Nikkels PG. Benign lesions of the vocal folds: histopathology and phonotrauma. Ann Otol Rhinol Laryngol. 1995 Sep;104(9 Pt 1):698-703.

14. Martins RH, Defaveri J, Domingues MA, de Albuguergue e Silva R. Vocal Polyps: clinical, morphological, and immunohistochemical aspects. J Voice. 2011 Jan; 25 (1): 98-106.

15. Bouchayer M, Cornut G. Microsurgical treatment of benign vocal folds lesions: indications, technique, results. Folia Phoniatr (Basel). 1992; 44 (3-4):155-84. 